\title{
O ENSINO DE PENSAMENTO COMPUTACIONAL ATRAVÉS DE UM JOGO DE TABULEIRO EM AMBIENTE DESPLUGADO: RELATO DE EXPERIÊNCIA DE FORMAÇÃO DOCENTE
}

\author{
Anderson Dall Agnol, IFRS Campus Porto Alegre, anderson.dallagnol@ifrs.edu.br \\ Crístian Gusberti, IFRS Campus Porto Alegre, crisgusberti@gmail.com \\ Silvia C. Bertagnolli, IFRS Campus Porto Alegre, silvia.bertagnolli@ poa.ifrs.edu.br
}

Resumo. Este artigo tem o objetivo de apresentar o processo de elaboração, aplicação e avaliação de um jogo de tabuleiro denominado Planetar, o qual tem por finalidade explorar de forma prática os quatro pilares do pensamento computacional (PC). Como metodologia, utilizou-se a pesquisa bibliográfica, a revisão sistemática sobre $\mathrm{PC}$ e um estudo de caso. Para o desenvolvimento do jogo foi adotado o processo de desenvolvimento de jogos educacionais chamado ENgAGED. O principal resultado identificado com a avaliação do jogo foi o aumento do interesse por parte dos participantes em ensinar PC na educação básica. Além disso, a execução do jogo também revelou que atividades lúdicas podem ser empregadas no engajamento de professores.

Palavras-chave: Pensamento Computacional, Jogos Desplugados, Jogo de Tabuleiro.

Teaching Computational Thinking through a Board Game in an Unplugged Environment: a teaching training experience report

Abstract. This article aims to present the process of elaboration, application, and evaluation of a board game called Planetar, which aims to explore in a practical way the four pillars of computational thinking. As methodology, bibliographic research, systematic review of computational thinking, and a case study were used. For the development of the game, the process of developing educational games called ENgAGED was adopted. The evaluation of the game identified a greater interest of the participants in teaching CT in basic education. Additionally, the execution of the game also revealed that playful activities can be used to engage teachers.

Keywords: computational thinking, digital information and communication technologies, unplugged games.

\section{Introdução}

A Computação passou a fazer parte do cotidiano das pessoas e organizações de diversos setores, permeando, inclusive, os ambientes educacionais. Segundo Menezes et al. (2018), os conhecimentos nessa área são muito importantes para a vida na sociedade contemporânea. Desta forma, torna-se fundamental que todos os indivíduos tenham conhecimentos básicos e possam aprender sobre Computação. O aprendizado dessa temática é importante, uma vez que problemas complexos de diferentes áreas da ciência estão sendo abordados sob uma perspectiva computacional (Brackmann, 2017).

Nesse contexto, é indispensável o ensino do Pensamento Computacional (PC) já na educação básica (Menezes, et al. 2018), pois para Kramer (2007) essa área envolve métodos para solucionar problemas com base em fundamentos, técnicas e estratégias da Ciência da Computação, tornando-se transversal às demais áreas do conhecimento. Percebe-se que tão importante quanto ensinar sobre essa temática é a necessidade de 
buscar estratégias e ferramentas que tornem o processo de ensino do PC mais lúdico e que despertem no estudante o engajamento e o interesse pelo assunto.

Nos últimos anos, diversas pesquisas apresentaram estratégias para incorporar o ensino de PC a outras disciplinas e temáticas, integrando ou não as Tecnologias Digitais da Informação e Comunicação (TDICs) no processo de ensino-aprendizagem. Por exemplo, os trabalhos de Silva et al. (2016) e Souza et al. (2019) trazem exemplos do ensino de PC com o apoio de jogos educacionais digitais e atividades com e sem uso de computadores (ou desplugadas). O trabalho de Pereira et al. (2019) descreve o processo de ensino-aprendizagem de PC usando atividades desconectadas aplicada em quatro turmas de uma escola pública do Ensino Fundamental II. Já Menezes et al. (2018) apresentam uma revisão bibliográfica detalhada que deixa claro que existem inúmeras outras abordagens plugadas e desplugadas para aplicar o PC em sala de aula, na educação básica.

Conforme argumentam Bell et al. (2009) é possível realizar atividades sobre conceitos computacionais sem o uso de computadores (computação desplugada). Essa é uma alternativa para as escolas públicas onde a infraestrutura é precária e são poucos os laboratórios de informática disponíveis, o mesmo é corroborado por (Unnikrishnan et al., 2016). O trabalho de Silva et al. (2014), vinculado a uma formação de professores, apontou um grande interesse dos docentes em utilizar a computação desplugada para introduzir o pensamento computacional em sala de aula.

Partindo-se desse contexto surgiu o problema de pesquisa que norteou esse trabalho: Como abordar o pensamento computacional de forma lúdica e desconectada, de modo a incentivar os professores da escola básica a incluí-lo em suas aulas? Assim, foi elaborado um jogo de tabuleiro denominado Planetar, com fabricação de baixo custo, o qual possui uma narrativa ampla, onde os conceitos de PC podem ser abordados através de suas mecânicas. Além disso, o jogo foi elaborado de modo a viabilizar a sua aplicação em escolas que não possuem computadores ou acesso à internet. O desenvolvimento do jogo foi fundamentado no processo de desenvolvimento de jogos educacionais chamado ENgAGED (EducatioNAl GamEs Development), o qual disponibiliza "suporte ao desenvolvimento sistemático de jogos educacionais" (Batistella; Wangenheim, 2016, p. 4).

O percurso metodológico selecionado foi composto por pesquisa bibliográfica, revisão sistemática sobre pensamento computacional desplugado, e por meio de um estudo de caso, no qual realizou-se a aplicação e a avaliação do jogo, usando um ambiente desconectado (sala de aula) com uma turma de estudantes do curso de Mestrado Profissional em Informática na Educação (MPIE), do Instituto Federal de Educação, Ciência e Tecnologia (IFRS) que tem como foco a formação de professores, sendo que a maioria deles já atua como docente na Educação Básica.

As próximas seções deste artigo apresentam aspectos teóricos relevantes sobre PC e jogos na educação (seção 2), os materiais e métodos utilizados na confecção e aplicação do jogo (seção 3), os resultados e discussões obtidos durante o processo (seção 4) e, por fim as considerações finais sobre a experiência na seção 5 .

\section{Pensamento Computacional e Jogos na Educação}

Com o advento do uso de fundamentos da computação para resolver problemas gerais, surge o conceito de pensamento computacional (Wing, 2006). Segundo Brackmann (2017), o pensamento computacional é uma capacidade criativa, crítica e estratégica do ser humano de fazer uso de fundamentos da Computação, nas mais diversas áreas do conhecimento para identificar e resolver desafios, de maneira individual ou colaborativa, com passos claros e simples, de maneira que uma pessoa ou uma máquina V. $18 \mathrm{~N}^{\circ} 1$, julho, 2020 RENOTE DOI: 
possam executá-los de maneira eficaz. Destaca-se que, para realizar atividades que envolvem o PC não é necessário que os indivíduos entendam de Computação.

O PC se estrutura sobre quatro pilares fundamentais (Brackmann, 2017; Wing, 2010): decomposição, reconhecimento de padrões, abstração e algoritmos. A decomposição envolve identificar um problema complexo e quebrá-lo em pedaços menores e mais fáceis de gerenciar. Esta atividade é essencial no desenvolvimento de algoritmos tratando de problemas complexos e é conhecida como "dividir para conquistar". Cada um desses fragmentos menores pode ser analisado individualmente com maior profundidade, identificando cenários parecidos que já foram solucionados anteriormente, reconhecendo desta forma padrões, focando apenas nos detalhes que são importantes, enquanto informações irrelevantes são ignoradas através da abstração, e por último, utilizando passos ou regras simples para resolver cada uma das partes encontradas, o que pode ser classificado como um algoritmo. É importante esclarecer que esses pilares não compreendem passos do PC, sendo assim, um ou alguns pilares podem ser empregados, não necessariamente existindo a obrigatoriedade de serem utilizados na sua totalidade ou sequencialmente. Nesse sentido, sob uma perspectiva educacional, o aprendizado e o desenvolvimento de fundamentos do PC, possibilitará aos estudantes adquirir uma habilidade indispensável que contribuirá para um melhor desempenho nas mais diversas disciplinas do currículo escolar (Glizt et al. 2017; Menezes et al. 2018).

O PC pode ser praticado através de jogos (Menezes et al. 2018), os quais compreendem um sistema onde os jogadores envolvem-se em um desafio abstrato, definido por regras, interatividade e feedback, podendo provocar reação emocional, e esta emoção pode estar relacionada com a ideia de diversão, até mesmo em um contexto educacional (Kapp, 2012). Prieto et al. (2005) explicam que os jogos que permeiam esses espaços devem possuir objetivos pedagógicos e sua utilização deve estar inserida em um cenário e em uma situação de ensino baseados em uma metodologia que oriente o processo, através da interação, da motivação e da descoberta, facilitando a aprendizagem de um conteúdo. Logo os jogos educativos podem auxiliar na solução de problemas e promover a aprendizagem, trazendo benefícios e desafios para a educação, como relatado nas pesquisas de Savi e Ulbricht (2008) e Santos e Isotani (2018).

Apesar do avanço tecnológico, muitas escolas não possuem infraestrutura adequada para utilizar jogos que usam recursos tecnológicos, desta forma, estratégias desplugadas constituem uma alternativa para abordar o tema em sala de aula. Segundo Sousa (2011), o qual introduziu conceitos básicos de computação através de um conjunto de atividades desplugadas em alunos do ensino médio, as tarefas que envolvem jogos fortalecem os laços de interação entre os usuários, reforçam a socialização, o diálogo e a troca de experiências.

Os jogos físicos de tabuleiro são recursos úteis no processo de ensino e aprendizagem desplugada em diversas disciplinas. O trabalho de Santos (2017), apresenta a elaboração, avaliação e uso de um jogo de tabuleiro para o ensino de artes, como também traz um modelo genérico dessa atividade para que professores possam adaptá-lo à sua realidade. Outro exemplo de jogo de tabuleiro é o Logirunner, idealizado por Casarotto et al. (2018) que visa reforçar os conteúdos de lógica e programação vistos em sala de aula através do emprego de desafios, uso de cartas, peças, tabuleiro e regras previamente definidas. Da mesma forma, o jogo aqui proposto foi desenvolvido no formato de um tabuleiro com cartas de desafios, que permitem que os personagens elaborados se desloquem pelas casas do tabuleiro. O uso de elementos estéticos e de design, de uma narrativa com temática que conduz ao espaço sideral, e o uso de regras foram pensados visando incentivar o engajamento, a motivação e a cooperação dos participantes da pesquisa. 


\section{Percurso Metodológico}

Inicialmente, foi realizada uma pesquisa bibliográfica sobre as principais teorias relativas ao pensamento computacional. Após, foi conduzida uma revisão da literatura considerando o uso dos seguintes termos: pensamento computacional desplugado e jogos, no Portal de Publicações da Comissão Especial de Informática na Educação (CEIE) e no Google acadêmico, que indexa publicações de diversas fontes, inclusive as que constam no Portal de Periódicos da Capes. Vários jogos foram encontrados, porém, o diferencial do jogo aqui descrito é que ele foi pensado como uma estratégia para mobilizar professores a adotarem o uso de pensamento computacional em sala de aula, tornando-o uma ferramenta formativa que instrui e incentiva esse público a ensinar PC em seu contexto profissional, adaptando ou recriando a proposta de acordo com sua disciplina, realidades e necessidades.

Após essa etapa, todo o planejamento e elaboração do jogo seguiram as fases estabelecidas pelo processo ENgAGED (Batistella; Wangenheim, 2016): fase 1 - análise da Unidade Instrucional (UI); fase 2 - projeto da UI; fase 3 - desenvolvimento do jogo educacional (seções 3.1 e 3.2); fase 4 - execução da unidade instrucional (seção 3.2); e a fase 5 - avaliação da UI (seção 4).

Ao finalizar o desenvolvimento do jogo realizou-se a sua aplicação e a avaliação, usando um ambiente desplugado. A coleta de dados foi conduzida durante a etapa de avaliação usando questionários compostos por perguntas abertas e fechadas, e também por um momento de reflexão do grande grupo, onde todo o processo foi avaliado. Ao concluir esse momento, a análise dos dados obtidos foi realizada, sendo que na seção 4 ela é sintetizada.

\subsection{Compreendendo o contexto}

A primeira fase do processo ENgAGED iniciou-se pela especificação do contexto da UI considerando o perfil dos aprendizes e o ambiente para, então, delimitar os objetivos de aprendizagem. Inicialmente, o público-alvo delimitado foram os professores da Educação Básica, que desejam abordar os pilares do pensamento computacional e adotar estratégias lúdicas em sala de aula. Isso porque, a realidade encontrada pela maioria desses docentes, em suas instituições de ensino, consiste na falta de acesso à internet, infraestrutura com poucos computadores ou inexistência de laboratórios de informática. Cabe destacar que, ao concluir o trabalho identificou-se que como muitas escolas possuem alguns jogos de tabuleiro concretos, e eles são usados em sala de aula, e com base na avaliação realizada pelos participantes percebeu-se que o público-alvo do jogo pode ser ampliado para estudantes também, pois essa é uma abordagem próxima da realidade encontrada nas escolas.

Com relação às competências referentes aos conceitos de pensamento computacional é necessário identificar os conhecimentos teóricos e práticos sobre o tema. O ensino do pensamento computacional em escolas é algo muito recente, com algumas práticas relatadas em eventos científicos, mas percebe-se pela análise dos trabalhos que muitos são conduzidos com alunos e poucos com docentes. Além disso, geralmente, o tema é abordado por docentes da área da matemática ou professores de apoio às tecnologias. A proposição deste trabalho consiste em demonstrar aos docentes de outras áreas que um jogo de tabuleiro pode contribuir para o desenvolvimento dessa temática em sala de aula, de modo a integrar diversos conhecimentos. 


\subsection{O projeto e o desenvolvimento do jogo Planetar}

Todo o jogo foi projetado visando explorar de forma prática os 4 pilares do pensamento computacional (decomposição, reconhecimento de padrões, abstração e algoritmos), de modo a complementar estudos teóricos sobre esses conceitos. Para tanto, foi selecionada como temática para o jogo o espaço sideral, bem como personagens fictícios - os ETs (extraterrestres). Esse cenário foi escolhido, porque ele pode ser compreendido como uma área de estudo interdisciplinar e se caracteriza como uma alternativa na abordagem do PC, uma vez que ele é frequentemente explorado em diferentes áreas do conhecimento. A partir do tema a narrativa concentra-se na história de alguns ETs que, percorrendo as galáxias que compõem o universo, ficaram perdidos e precisam de ajuda para resolver alguns enigmas do percurso do tabuleiro e retornar para casa. Esse retorno ao lar motiva a organização do jogo no formato de tabuleiro, onde as casas devem ser percorridas através da resolução de problemas relacionados aos 4 pilares do PC. Essa narrativa tem como foco incentivar a imersão do jogador (Batistella; Wangenheim, 2016).

O tabuleiro foi desenhado em um software de edição e diagramação de imagens, e foi impresso em uma impressora plotter, colado e plastificado sobre uma placa de MDF de $60 \times 40 \mathrm{~cm}$. O tabuleiro foi modelado tendo como definição principal o quantitativo de 20 casas, dessas, cinco contendo desafios lógicos para que a atividade fosse realizada em aproximadamente 2 períodos de aula. A Figura 1 à esquerda ilustra o projeto original do tabuleiro, depois do teste realizado o tabuleiro foi redesenhado (Figura 1, à direita).
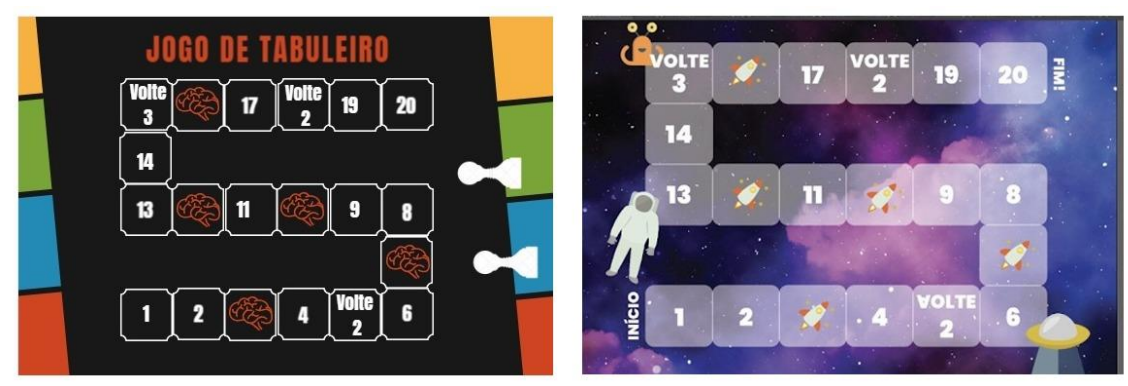

Figura 1 - Tabuleiro original (esquerda) e finalizado (direita) Fonte: Elaborado pelos autores, 2019.

Os personagens principais do jogo são ETs que assumiram cores diversificadas (vermelho, branco, azul e preto) inspiradas em planetas, galáxias, estrelas, corpos celestes e outros elementos espaciais. Cada personagem é a representação físicas das equipes de jogadores no tabuleiro, como ilustra a Figura 2. Inicialmente, foram criados 4 personagens iniciais, mas o jogo pode ser ampliado introduzindo outros usando cores diferenciadas.
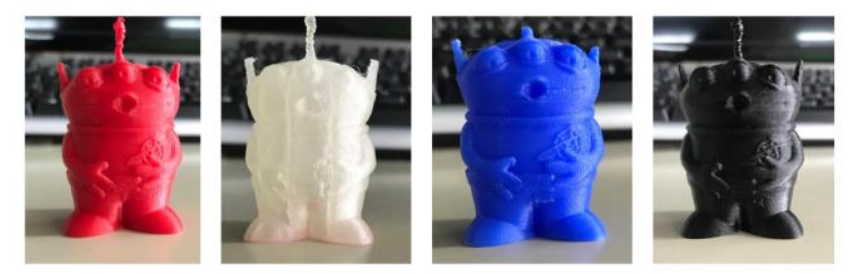

Figura 2 - Os Personagens do Planetar Fonte: Elaborado pelos autores, 2019.

Para percorrer o tabuleiro foram usados dois dados: um dado tradicional com 6 faces numéricas, que permite percorrer as casas do tabuleiro, e outro dado com as faces nas seguintes cores: vermelho, branco, azul e preto, que correspondem às cores dos ETs, e duas faces nulas, que indicam a necessidade de jogar o dado colorido novamente. Observa-se que tanto os personagens quanto os dados utilizados no jogo foram criados a 
partir de modelos 3D disponíveis nas bases de dados Thingiverse ${ }^{1}$ e Cults $3 \mathrm{D}^{2}$, sendo que esses elementos foram confeccionados em uma impressora 3D. No caso de escolas públicas que não possuem acesso a este tipo de recurso, a confecção do tabuleiro poderia ser realizada em aulas de artes com pintura em cartolina, EVA ou papelão; já os personagens poderiam ser confeccionados de sucata ou com materiais reciclados.

Na sequência, partiu-se para a seleção e confecção dos desafios do jogo. Eles foram compostos por problemas simples de lógica que precisavam ser resolvidos pelos jogadores nas casas destinadas aos desafios. Esses problemas foram agrupados em 5 níveis crescentes de dificuldade, representados por cartas em diferentes cores (verde, amarelo, azul, laranja e vermelho, respectivamente). As cartas contendo os desafios foram elaboradas no sentido de garantir que em cada conjunto de cores existisse ao menos um desafio relacionado a cada um dos 4 pilares do PC. A Figura 3 exemplifica 4 dos 30 desafios presentes no jogo.
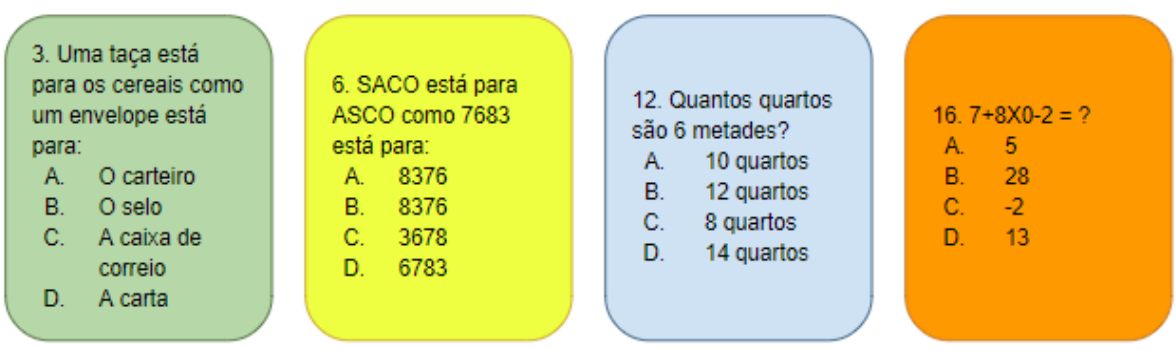

Figura 3 - Exemplos de cartões de desafios Fonte: Elaborado pelos autores, 2019.

Ao finalizar o projeto do jogo ele foi testado pelos pesquisadores, onde suas regras e mecânicas foram analisadas, bem como o tempo de jogo, visando identificar se ele era factível de ser jogado no prazo estimado. Isso foi feito, porque conforme estabelecem Battistella e Wangenheim (2016, p. 382) "Os testes são realizados pelos criadores do jogo [...] permitindo assim testar os níveis e funcionalidades do jogo, além de verificar os possíveis problemas antes de sua execução (Fase 4)".

A execução do jogo compreendeu a organização dos participantes em 4 equipes compostas por 3-4 jogadores. Em seguida foram lidas as regras do jogo explicando que: a ordem das jogadas seria definida por um sorteio inicial com o dado colorido com as faces representando as cores dos personagens. As jogadas consistiam em andar o número de casas sorteadas no dado numérico, caso o número sorteado fosse 6 a equipe perderia a vez em duas rodadas consecutivas. Ao parar em um desafio o grupo pescaria uma carta correspondente ao seu nível de dificuldade e que as cartas estariam posicionadas em pilhas, separadas por cores. Todos os grupos contaram com até 3 minutos para resolver o desafio, mas somente a equipe que fez a jogada poderia tentar responder. Caso a resposta da equipe não fosse a correta, a equipe perderia a vez na próxima jogada, e então o dado colorido seria jogado e a cor sorteada definiria a cor da equipe que passaria a tentar responder. Se a equipe sorteada errasse não perderia a vez de jogar e se acertasse poderia andar o número de casas sorteado pelo dado da equipe anterior.

Após as etapas de construção o jogo foi avaliado sob dois aspectos principais: avaliação da aprendizagem e avaliação sob o ponto de vista da experiência do jogador. Conforme definem Battistella e Wangenheim (2016) a avaliação deve ser conduzida após a execução do jogo "utilizando o instrumento para coleta de dados definidos". Os dados obtidos com esse processo de avaliação encontram-se descritos na próxima seção.

\footnotetext{
${ }^{1}$ Disponível em: https://www.thingiverse.com/ e ${ }^{2}$ Disponível em: https://cults3d.com/en V. $18 \mathrm{~N}^{\mathrm{o}} 1$, julho, 2020 DOI:
} 


\section{Resultados e Discussões}

Para a avaliação da aprendizagem foi utilizada a teoria de Ausubel (1982). Segundo ele para determinar se a aprendizagem foi significativa é necessário considerar e avaliar os saberes pré-existentes no sujeito, como também verificar o que foi assimilado por eles após a exposição de conceitos e conteúdo. A teoria da aprendizagem significativa (Ausubel, 1982) estabelece que todo novo conhecimento é ancorado nos conhecimentos prévios do sujeito. Assim para verificar a efetividade do jogo como ferramenta para a formação dos docentes foram elaborados dois questionários para a avaliação da aprendizagem: um pré-teste e um pós-teste. Ambos continham perguntas relacionadas aos principais conceitos de PC e sua utilização no cotidiano, bem como na resolução de problemas.

O questionário pré-teste foi aplicado com os 12 participantes antes da aplicação do jogo. Partindo-se do pressuposto que os jogadores não possuem qualquer conhecimento do conteúdo a ser avaliado considerou-se relevante identificar se os participantes poderiam distinguir em que situações o PC poderia ser aplicado ou se conseguiam apontar a utilização desta técnica na resolução de problemas do seu cotidiano. O questionário pós-teste foi aplicado junto aos jogadores após a execução do jogo.

Tanto no pré-teste como no pós-teste os participantes foram questionados considerando-se 7 dimensões: conceitos básicos de PC e sua aplicabilidade na resolução de problemas; o pensamento computacional e o cotidiano; a decomposição; a identificação de padrões; a abstração; os algoritmos; e se os participantes conseguiam identificar os conceitos de PC e os relacionar com a importância do seu ensino na educação básica. Examinando todas as respostas obtidas para essas dimensões a que apresentava relação com a aplicabilidade do PC na educação básica revelou o maior percentual de divergência, conforme ilustra o Gráfico 1.

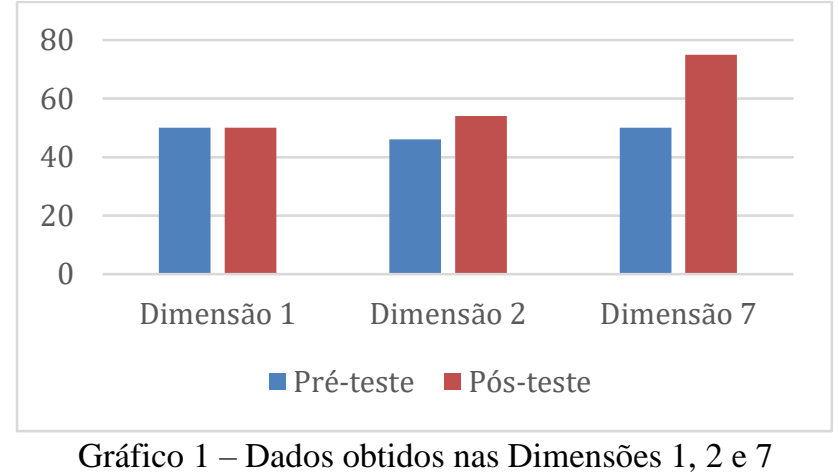

No momento final de avaliação do jogo, foi realizada uma reflexão sobre ele e seu impacto na aprendizagem, os participantes relataram que essa seria uma estratégia diferenciada para ensinar técnicas de pensamento computacional na educação básica, sendo que alguns argumentaram que por usar jogos de tabuleiro com seus alunos o conteúdo parecia ser mais simples de compreender. Com relação às competências referentes aos conceitos de pensamento computacional, alguns já os conheciam, e apenas um havia experienciado com seus alunos essa temática na prática. Porém, mesmo relatando no pré-teste que já conheciam o PC quando arguidos sobre os pilares que faziam parte dos desafios, constantes nas cartas do jogo, alguns não sabiam responder com precisão.

Quando o jogo começou a ser projetado algumas inquietações dos pesquisadores foram debatidas: a interdisciplinaridade e a importância do trabalho em grupo. Então, o jogo foi projetado seguindo uma temática que permitisse explorar a interdisciplinaridade; e adotando uma estrutura que viabilizasse que fosse jogado em equipe. Isso porque, 
esperava-se que os participantes trocassem experiências para solucionar o problema de forma conjunta. Um dos pontos principais do jogo seria viabilizar um tempo para as interações entre os participantes de uma mesma equipe, então no teste do jogo foi estimado um tempo para que as discussões e trocas de saberes ocorressem. Conforme argumenta Moita (2007, p. 8) “... o conhecimento resulta de um processo de exploração, experimentação, discussão e reflexão colaborativa realizado não só de forma ativa pelo aprendiz, mas também no grupo...". Com relação a isso, quando os participantes foram indagados no momento de reflexão final eles apontaram que essas trocas permitiram consolidar alguns conhecimentos sobre os quatro pilares do PC, identificar os passos que alguns membros da equipe usavam para resolver os problemas (pilar algoritmo), as analogias realizadas (pilar reconhecimento de padrões) e como os problemas eram quebrados para se chegar ao resultado final (pilar decomposição).

Em relação à avaliação do jogo sob o ponto de vista da experiência do jogador, todos os participantes consideram o design, a interface, a narrativa e demais elementos bastante atrativos, enfatizando que o jogo poderia ser adaptado a diferentes contextos educacionais. Sobre o formato de jogo de tabuleiro em ambiente desplugado, todos afirmaram ser adequado e mencionaram que esse modelo favorece a atenção e o engajamento. Perguntados sobre o sentimento de competição e cooperação todos afirmam ter notado a presença de ambos, alegando inclusive que esses são saudáveis e próprios a todos os jogos educacionais. Por fim, quando foram questionados sobre possíveis mudanças para próximas aplicações os participantes relataram apenas melhorias em aspectos estéticos (impressão dos dados e cartas) e não expuseram nenhuma alteração que influencie na aplicação da atividade.

Embora os jogadores estivessem utilizando pilares do PC para resolver todos os desafios, acredita-se que eles não perceberam tal aplicação por estarem bastante imersos na atividade focando em aspectos do jogo (game), como o ambiente de cooperação, a competição, a narrativa, as regras e aos aspectos visuais. Convém destacar que, no momento de reflexão, dois participantes argumentaram que os pilares do PC estavam presentes inclusive na organização do tabuleiro e nas regras que permitiam percorrê-lo. Eles apontaram que todos esses elementos tornaram a atividade mais divertida, com potencial de ser adaptável a diferentes contextos educacionais. Acredita-se que seja necessário aplicar e avaliar o jogo novamente, junto a um número maior de participantes, visando coletar mais dados e estabelecer comparações com este primeiro estudo de caso.

\section{Considerações Finais}

O presente artigo apresentou o projeto, a execução e a avaliação do jogo de tabuleiro Planetar que busca abordar os conceitos de PC em um ambiente desplugado. Os dados utilizados nesta pesquisa foram coletados através de três questionários, dois vinculados à aprendizagem (pré-teste e pós-teste) que possibilitaram identificar que do ponto de vista conceitual os participantes haviam compreendido os quatro pilares, mas que ao colocar os conceitos em prática a aquisição do conhecimento não se refletia no mesmo percentual. $\mathrm{O}$ terceiro questionário que possibilitou investigar como foi a experiência do jogador, suas percepções sobre o jogo (estética e recursos visuais).

Embora os questionários tenham apontado vários elementos que foram essenciais para a avaliação do jogo, foi no momento de reflexão que os participantes relataram aspectos e situações que não estavam previstos nos questionários, por exemplo, a estrutura do tabuleiro fundamenta-se nos pilares do PC, existem várias situações do cotidiano em que os pilares do pensamento computacional podem ser aplicados, em especial os algoritmos, e que o PC não é um conceito complexo, como alguns acreditavam ser antes da experiência conduzida com os participantes. 
Por fim, após a execução de todo o trabalho pode-se perceber que o estilo de jogo de tabuleiro é eficiente para explorar os conceitos básicos de pensamento computacional, e é uma alternativa viável para professores de escolas públicas que não possuem os recursos tecnológicos adequados, mas que mesmo assim desejam diversificar suas práticas pedagógicas.

\section{Referências}

AUSUBEL, D. P. A aprendizagem significativa: a teoria de David Ausubel. São Paulo: Moraes, v. 198, 1982.

BATTISTEllA, P. E.; WANGENHEIM, C. G. v. ENgAGED: Um Processo de Desenvolvimento de Jogos para Ensinar Computação. Anais... Simpósio Brasileiro de Informática na Educação. p. 380-389, 2016. Anais. Disponível em: <https://www.brie.org/pub/index.php/sbie/article/view/6718/4606> Acesso em: 10 mar. 2019.

BELL, T.; ALEXANDER, J.; FREEMAN, I.; GRIMLEY, M. Computer science unplugged: School students doing real computing without computers. The New Zealand Journal of Applied Computing and Information Technology, v. 13, n. 1, p. 20-29, 2009.

BRACKMANN, C. P. Desenvolvimento do pensamento computacional através de atividades desplugadas na educação básica. 2017. Tese de doutorado. Disponível em: <https://www.lume.ufrgs.br/handle/10183/172208> Acesso em: 14 nov. 2018.

CASAROTTO, I. R. et al. Logirunner: um Jogo de Tabuleiro como Ferramenta para o Auxílio do Ensino e Aprendizagem de Algoritmos e Lógica de Programação. RENOTE, v. 16, n. 1, p.1-10, 2018. Disponível em: 〈https://seer.ufrgs.br/renote/article/view/85998> Acesso em: 05 maio 2020.

GLIZT, F. R. O.; et al. O Pensamento Computacional nos Anos Iniciais do Ensino Fundamental. RENOTE, v. 15, n. 2, 2017. Disponível em: <https://seer.ufrgs.br/renote/article/view/79226/46118> Acesso em: 10 de nov. de 2018.

KAPP, K. M. The gamification of learning and instruction: Game-based methods and strategies for training and education. San Francisco: Pfeiffer, 2012.

KRAMER, J. Is abstraction the key to computing?. Communications of the ACM, v. 50, n. 4, p. 36-42, 2007. Disponível em: <https://dl.acm.org/doi/pdf/10.1145/1232743.1232745> Acesso em: 10 de nov. 2018.

MENEZES, C. S.; NUNES, D.; LIVI, M. A. C. Pensamento Computacional: Revisão bibliográfica. Porto Alegre: UFRGS, 2018. Disponível em: <https://lume.ufrgs.br/handle/10183/197566> Acesso em: 03 out. 2019.

MOITA, F. M. G. S. C.; SILVA, A. C. R. Os games no contexto de currículo e aprendizagens colaborativas online. In: SILVA, E. M; SOUZA, R. P. (Org). Jogos eletrônicos - Construindo novas trilhas. Campina Grande: EDUEP, p. 45-52, 2017.

PEREIRA, F. T. S. S.; ARAÚJO, L. G. J.; BITTENCOURT, R. A. Intervenções de Pensamento Computacional na Educação Básica através de Computação Desplugada. In: Workshop de Informática na Escola. p. 315-324, 2019. Anais. Disponível em: <https://www.br-ie.org/pub/index.php/wie/article/view/8518/6091> Acesso em: 10 mar. 2020 .

PRIETO, L. M.; TREVISAN, M. C. B.; DANESI, M. I.; FALKEMBACH, G. A. M. Uso das Tecnologias Digitais em Atividades Didáticas nas Séries Iniciais. Revista Novas V. $18 \mathrm{~N}^{\mathrm{o}} 1$, julho, 2020 RENOTE DOI: 
Tecnologias na Educação, v. 3, n. 1, p.1-11, maio 2005. Disponível em: <http://www.cinted.ufrgs.br/renoteold/maio2005/artigos/a6_seriesiniciais_revisado.pdf> Acesso em: 05 maio 2020.

SANTOS, M. S. Jogo de Tabuleiro Educacional: De um jogo para o ensino de artes a um modelo genérico para criação de múltiplos jogos. Porto Alegre, IFRS, 2017. Dissertação de mestrado. Disponível em: <http://atom.poa.ifrs.edu.br/uploads/r/biblioteca-clovis-vergara-marques4/1/6/8/16805d283ee4140258a69b476b8ca1723022f6e4ae8a6b6a9e66ec73b2a8ffcf/Dis serta__o_FINAL_Miria.pdf> Acesso em: 05 maio 2020.

SANTOS, W. O.; ISOTANI, S. Desenvolvimento de Jogos Educativos? Desafios Oportunidades e Direcionamentos de Pesquisa. RENOTE, Porto Alegre, v. 16, n. 2, p.110, dezembro 2018. Disponível em: <https://seer.ufrgs.br/renote/article/view/89252/51492> Acesso em: 05 maio 2020.

SAVI, R.; ULBRICHT, V. R. Jogos Digitais Educacionais: Benefícios e Desafios. RENOTE, Porto Alegre, v. 6, n. 2, p.1-10, dezembro 2008. Disponível em: <https://seer.ufrgs.br/renote/article/view/14405/8310> Acesso em: 05 maio 2020.

SILVA, V.; SOUZA, A.; MORAIS, D. Pensamento Computacional: Um relato de práticas pedagógicas para o ensino de computação em escolas públicas. Revista Tecnologias na Educação. In: Congresso Regional sobre Tecnologias na Educação. Anais. ano 8, v.16. Disponível em: <http://tecedu.pro.br/wpcontent/uploads/2016/09/Art5-Pensamento-computacional-Um-relato-depr\%C3\%A1ticas-pedag\%C3\%B3gicas....pdf> Acesso em: 01 maio 2018.

SILVA, T. R.; ARAÚJO, G. G.; ARANHA, E. H. S. Oficinas Itinerantes de Scratch e Computação Desplugada para Professores como apoio ao Ensino de Computação - um Relato de Experiência. In: Workshop de Informática na Escola. p. 380-389, 2014. Anais. Disponível em: $<$ https://www.brie.org/pub/index.php/wie/article/view/3121/2629> Acesso em: 10 mar. 2020.

SOUZA, D. S.; DIAS, J.; SANTOS, K. S. "Não ligue o computador": a Computação Desplugada como Estratégia Metodológica para o desenvolvimento do Pensamento Computacional na Educação Básica-Uma Revisão Sistemática da Literatura. RENOTE, v. 17, n. 3, 2019. Disponível em: <https://seer.ufrgs.br/renote/article/view/99526/55670> Acesso em: 30 abr. 2020.

SOUSA, R. V. et al. Ensinando e aprendendo conceitos sobre ciência da computação sem o uso do computador: Computação Unplugged!!!. In: Jornada de Atualização em Informática na Educação, v. 1, n. 1, 2011. Anais. Disponível em: <http://www.brie.org/pub/index.php/pie/article/view/1305> Acesso em: 15 nov. 2018.

UNNIKRISHNAN, R.; AMRITA, N.; MUIR, A.; RAO, B. Of Elephants and Nested Loops: How to Introduce Computing to Youth in Rural India. Proceedings... International Conference on Interaction Design and Children, Manchester, UK, p. 137-146, 2016.

WING, Jeannette M. Computational thinking. Communications of the ACM, v. 49, n. 3, 2006, p. 33-35. 20 Disponível <https://dl.acm.org/doi/fullHtml/10.1145/1118178.1118215> Acesso em: 04 nov. 2018

WING, Jeannette M. Computational Thinking: What and Why?. 17. out. 2010. Disponível em <http://www.cs.cmu.edu/ CompThink/resources/TheLinkWing.pdf> Acesso em: $01 \mathrm{dez} 2018$. 